\title{
Feed Passage in Broilers - A Complex Problem ${ }^{1}$
}

\author{
G. D. Butcher, DVM, Ph.D., A. H. Nilipour, Ph.D., R. D. Miles, Ph.D. ${ }^{2}$
}

"Feed passage," or, more accurately, the passage of undigested feed in the feces of broilers, continues to be reported in broiler integrations on a sporadic basis. The incidence and severity are much lower at present than during 1996 when global reports of feed passage occurred following consecutive years of poor grain harvests. At that time, broilers were passing undigested feed in the feces and this was evidenced by the presence of large corn particles, excess moisture, a characteristic green coloration with orange mucus, and poor formation to the feces. Affected broilers had poor pigmentation, poor feed conversion, and lower body weights, and flock uniformity was variable. On necropsy, lesions were commonly found in the proventriculus and gizzard of affected broilers. Lesions were also found in the small intestines. It was interesting to note that in countries such as Guyana where alternative grains such as rice were fed, this syndrome did not occur.

Intensive investigations conducted during the 1996 global feed passage problem failed to identify any specific toxin in the grains to account for this widespread syndrome. It was widely believed that the disease was related to some unidentified toxin and/or that the nutrient content of the grain was poor due to the low quality of available grain. The severity and clinical manifestations of the problem varied among integrations depending on management practices, presence of concurrent diseases, and specific shipments of grain.

According to recent reports, feed passage in broilers is occurring in individual farms or, in some cases, in entire integrations. The disease is not being observed on a global basis as in the past. The tendency for broiler managers, from past experiences, is to immediately blame the feed quality. However, the lack of consistency of the problem in farms suggests other factors may need to be considered.

Following is a summary of factors that should be considered when passage of undigested feed is noted in broilers. This summary is by no means complete, as additional etiologies will continue to be identified and added to the list. Feed passage syndrome in broilers, for purposes of this paper, will be defined as "the passage of nutrients in feces due to improper digestion and/or absorption in the intestines." Note that the cause of the syndome could be the broiler's inability to digest the feed or to absorb the feed, or both. It should be understood when investigating the cause(s), that passage of undigested nutrients in the feces may be resulting from a single factor or a combination of factors.

1. This document is VM123, one of a series of the Veterinary Medicine-Large Animal Clinical Sciences Department, Florida Cooperative Extension Service, Institute of Food and Agricultural Sciences, University of Florida. Original publication date May 1, 2002. Visit the EDIS Web Site at http://edis.ifas.ufl.edu.

2. Gary D. Butcher, extension veterinarian, University of Florida College of Veterinary Medicine, Gainesville, FL., Amir H. Nilipour, PhD, Director of Investigation and Quality Assurance, Grupo Melo, S.A., Panama, Republic of Panama 
Many infectious and nutritional diseases cause primary lesions in the gut. For example, avian influenza, Newcastle disease, vitamin A deficiency, and histomoniasis are all capable of producing such changes. Influenza and Newcastle cause enteritis with localized hemorrhage and necrosis.

Histomoniasis is often associated with severe lesions in the ceca. Vitamin A deficiency causes lesions in the crop and esophagus. It should also be recognized that most infectious diseases cause at least a transitory diarrhea or passage of feces with excess moisture. During the early stages of most infectious diseases, such as infectious bronchitis, infectious laryngotracheitis, avian encephalomyelitis, etc., birds experience diarrhea. No gross lesions are present in the gut associated with these infections.

Clearly, the diseases described above are much different than the classical feed passage syndrome and include diarrhea as only a part of their clinical expression. This information was presented simply to suggest that feed passage is a general term and is not associated with a specific disease syndrome, but occurs during many diseases. Feed passage results from some abnormality in the digestion and absorption of nutrients, and although the causes may be numerous, the clinical expression in the broiler is simply feed passage.

\section{Causes of Feed Passage Syndrome}

\section{Heat Stress}

When broilers are heat stressed, they increase consumption of water in an effort to cool down. Much of this water will be voided in the feces, resulting in wet and poorly formed feces. Following heat stress, physical changes in the intestinal lining can be detected by histologic study within 48 hours and can be observed for at least 3 days. Changes include a decrease in villus length and surface area.

\section{Dietary Salt Intake}

When salt consumption is increased, broilers will consume additional water to assist in the elimination of the salt. This will result in broilers voiding much of the excess water by way of the feces. The result is wet and poorly formed feces. Excess salt intake can result from mixing errors in the feed, not taking into consideration the salt levels in some ingredients such as fishmeal when formulating, or not considering the salt levels in the drinking water. It must be understood that if drinking water is slightly brackish, this salt intake must also be taken into consideration when formulating the feed. During hot periods, producers may stimulate water consumption in poultry by increasing salt levels in the feed.

\section{Coccidiosis}

Damage to the lining of the intestine from clinical and subclinical coccidiosis can cause passage of undigested nutrients in the feces, especially in younger broilers. Feed passage is usually associated with the species, E. acervulina and E. maxima. A coccidiosis monitoring program combined with an effective coccidiostat control program are recommended to ensure coccidiosis is not damaging the lining of the intestine and affecting the broiler's ability to absorb nutrients.

\section{Ascarids and Cestodes}

Intestinal parasites can irritate the gut and cause passage of undigested feed in the feces. A mild infestation of ascarids is enough to cause irritation and feed passage, while a significant cestode infection must occur before losses are recognized.

\section{Viral Infections}

A number of viruses are associated with broiler feed passage. These include reovirus, calicivirus, adenovirus, parvovirus, enterovirus, coronavirus, togavirus, and others. Historically, reovirus has been considered a primary viral cause of broiler feed passage or "malabsorbtion syndrome." However, it is now clear that this virus does not play a significant role in feed passage in broilers. Some investigators have described this virus as "one in search of a disease" as it is wrongly blamed for many syndromes in poultry. Reovirus infection is responsible for the disease viral arthritis and vaccination to control this disease is recommended.

\section{Bacterial Infections}

Bacterial enteritis often occurs secondary to viral or coccidial infections. Prevention is best achieved with effective use of growth promoters, control of 
coccidial infections, protection of immune system integrity, and comprehensive biosecurity and sanitation practices. The intestinal tract houses a large bacterial population that is necessary for its normal function. An imbalance in this flora can result in disease.

A specific bacterial infection in the gut that is increasing in incidence in recent years is necrotic Enteritis, cause by $C$. perfringens. This bacterium is common in the environment and is considered part of the normal gut flora of poultry. However, disease associated with this bacterium is occurring with increasing frequency in recent years. A number of predisposing factors are recognized, including 1) damage to the gut from coccidiosis, 2) exposure to extremely high levels of the organism in the poultry house environment, 3) dietary changes including $\mathrm{pH}$ and viscosity, 4) high energy diets, 5) restricted feeding programs, 6) overstocking or high density, 7) saturation of the litter or poor litter management, 8) changes in the physical form of the feed (mash to pellets) during the grow-out, and 9) feeding of some animal proteins.

A scenario for the development of necrotic enteritis follows: An infection with E. acervulina causes damage to the gut lining. The damage causes reduction in the intestinal passage of ingesta. This permits an overgrowth of $C$. perfringens due to the decrease of the natural "flushing" of the gut contents. Necrotic enteritis occurs.

The significant increase in the incidence of necrotic enteritis is of concern for many producers. In some areas, it was shown that the soil level of the bacterium is extremely high ( $>600,000$ spores per gram of soil) when conditions are ideal, such as moisture, $\mathrm{pH}$, etc. Treatment of such premises should include a complete clean out and application of salt at a rate of 60 pounds per 1000 square feet. Following 2 or 3 days when the salt has had a chance to dissolve into the soil, the litter is replaced. High salt levels provide an adverse environment for the bacterium and the numbers will be reduced drastically. This soil treatment may need to be repeated.

\section{Mycotoxins}

More than 200 feed-related mycotoxins have been identified. Common examples include Ochratoxin A, which causes increased intestinal fragility and decreased shear strength. Aflatoxin causes liver damage, blockage of bile ducts, and decreased level of bile in the lower intestine. A poor fat absorption would result. Trichothecene (T-2) is associated with lesions in the oral cavity, proventriculus, gizzard, and intestines. This mycotoxin is very caustic and causes chemical burns to the sites that it contacts.

Contamination sources for mycotoxins include moldy grain, dirty feed bins, and contaminated feed transfer equipment. It is important to prevent growth of mold in feed by purchasing only quality grains and storing grains under suitable conditions. Inhibitors can be added to grains to restrict growth of molds. However, grains with higher levels of mycotoxins should have binding agents added and be blended with better quality grains to reduce negative effects.

\section{Tannins}

Toxic levels of tannins in the feed cause esophageal and gastric edema, hemorrhagic ulceration, necrosis and sloughing of the mucosal lining, hypersecretion of mucin, and crop wall thickening. This damage results in feed passage. Tannic acid is found in the coatings of grains, especially some varieties of sorghum. The presence of tannin in grains is of benefit to grain farmers in that higher levels will reduce consumption of the grains by wild birds during production in the field. Of course, they will also have the effect of causing poor grain palatability and feed refusal in commercial poultry when levels are elevated.

\section{Biogenic Amines}

These compounds are present in low levels in animals, plants, and microorganisms. At higher concentrations they are toxic. Biogenic amines cause more problems in hot seasons when there is increased bacterial degradation of free amino acids, dietary proteins, and animal by-products. 
Lesions associated with feeding toxic levels of biogenic amines include proventricular enlargement, gizzard erosion, sloughing of intestinal epithelia, poor weight gain and feed conversion, impaired immune response, and diarrhea. Affected birds are poorly pigmented and have low body weights and feed conversions.

Table 1.

\begin{tabular}{|l|l|}
\hline Amino Acid & Biogenic Amine \\
\hline Histadine & Histamine \\
\hline Arginine/ornithine & Putrecine \\
\hline Lysine & Cadaverine \\
\hline Methionine & Spermadine \\
\hline Tyrosine & Tyramine \\
\hline Phenylalinine & Phenylethylamine \\
\hline
\end{tabular}

\section{Gizzerozine}

This toxin is produced in over-processed fishmeal. It has an effect similar to the biogenic amine, histamine, in causing overproduction of hydrochloric acid in the proventriculus and resulting gizzard erosion. Toxicity is prevented through feeding only quality, properly processed fishmeal.

\section{Rancid Fats}

Feeding of rancid fats to broilers is a common cause of feed passage, especially in hotter climates where fats readily become rancid if not stored properly. Lesions include proventriculitis, gizzard erosions, and enteritis. The process involves the oxidizing of dietary ingredient fat and fat-soluble compound to produce free radicals or reactive oxygen molecules. Examples include the surperoxide radical $\left(\mathrm{O}_{2}\right)$, hydrogen peroxide $\left(\mathrm{H}_{2} \mathrm{O}_{2}\right)$, and the hydroxyl radical (HO-). These compounds cause impaired immunity, poor growth, poor feed conversion, poor pigmentation, and gizzard erosion.

\section{Water Quality}

The composition of a broiler's body is $60 \%$ water. It is essential that broilers drink adequate quantities of water in order to meet body demands. In many integrations, ensuring water quality is not considered a priority and consumption of poor quality water causes irritation of the gut and inefficient nutrient absorption leading to rapid feed passage. In order to reduce the affects of bacterial contamination, it is very important to chlorinate the water so 1-3 PPM free chlorine is present at the level consumed by the birds. The use of closed type drinkers is strongly recommended. If bell shape drinkers are used, they should be regularly cleaned and disinfected. The older, trough-type drinker is least desirable because it spills constantly and is easily contaminated.

\section{Litter Quality}

Litter is the first material that recently placed chicks might consume, before finding feed and water. Therefore it is important to ensure litter is high quality, free from foreign materials, and fresh. If quality litter is not available for the new flock, at least provide quality litter in the brooding area during the first week. Ingestion of contaminated litter can cause irritation of the gut linings of the young chick, resulting in poor nutrient absorption.

\section{Conclusion}

Identifying the specific cause(s) of feed passage in an integration may be difficult, as many complex factors need to be considered. In some cases, several factors work in concert and limit the broiler's ability to digest and/or absorb feed, resulting in the passage of undigested nutrients. Investigation of the cause(s) requires gross examination of the affected poultry, examination for presence of intestinal parasites, bacterial culture, virus isolation, histopathlogy, and toxicological testing. These diagnostic capabilities are not readily available to many integrations; thus identifying the cause of the problem often is based on more subjective observations. It is clear that management practices can play a major role in preventing this problem. Feed passage directly affects the most important broiler economic performance parameters, feed conversion and body weight. When feed passage is observed in broiler houses, evaluating basic management practices may be the most efficient manner to resolve the problem.

Feed passage in poultry will continue to be an occasional problem in poultry integrations. It is important to keep an open mind, as many factors 
need to be considered when investigating the problem. 